\title{
High-resolution Fourier-transform spectrometer chip with microphotonic silicon spiral waveguides
}

\author{
Aitor V. Velasco, ${ }^{1,3}$ Pavel Cheben, ${ }^{2, *}$ Przemek J. Bock, ${ }^{2}$ André Delâge, ${ }^{2}$ Jens H. Schmid, ${ }^{2}$ Jean Lapointe, ${ }^{2}$ \\ Siegfried Janz, ${ }^{2}$ María L. Calvo, ${ }^{1}$ Dan-Xia Xu, ${ }^{2}$ Mirosław Florjańczyk, ${ }^{2}$ and Martin Vachon ${ }^{2}$ \\ ${ }^{1}$ Departamento de Óptica, Facultad de Ciencias Físicas, Universidad Complutense de Madrid, Madrid 28040, Spain \\ ${ }^{2}$ National Research Council Canada, Ottawa, Ontario K1A oR6, Canada \\ ${ }^{3}$ e-mail: avillafr@pdi.ucm.es \\ ${ }^{*}$ Corresponding author: pavel.cheben@nrc.ca
}

Received December 21, 2012; revised January 25, 2013; accepted January 25, 2013;

posted January 29, 2013 (Doc. ID 182206); published February 25, 2013

\begin{abstract}
We report a stationary Fourier-transform spectrometer chip implemented in silicon microphotonic waveguides. The device comprises an array of 32 Mach-Zehnder interferometers (MZIs) with linearly increasing optical path delays between the MZI arms across the array. The optical delays are achieved by using Si-wire waveguides arranged in tightly coiled spirals with a compact device footprint of $12 \mathrm{~mm}^{2}$. Spectral retrieval is demonstrated in a single measurement of the stationary spatial interferogram formed at the output waveguides of the array, with a wavelength resolution of $40 \mathrm{pm}$ within a free spectral range of $0.75 \mathrm{~nm}$. The phase and amplitude errors arising from fabrication imperfections are compensated using a transformation matrix spectral retrieval algorithm. (c) 2013 Optical Society of America
\end{abstract}

OCIS codes: $130.3120,300.6300$

Compact spectral filters with a small footprint are required for a wide range of applications, including wavelengthdivision-multiplexed communications, optical interconnects, biological and environmental sensing, and space instrumentation [1]. Planar waveguide devices such as arrayed waveguide gratings (AWGs) [2], echelle gratings $[3,4]$, lattice filters [్], ring resonators []], and sidewall grating filters $[7,8]$ can achieve a high spectral resolution for a small device footprint, but their optical throughput (étendue) is largely limited by the requirement of a singlemode input waveguide. Étendue is of critical importance for spectroscopy applications, especially when spatially extended and incoherent sources are analyzed. This limitation can be overcome with planar waveguide Fouriertransform (FT) spectrometers $[\underline{9,10]}$ based on the principle of spatial heterodyne spectroscopy (SHS) [11]. SHS benefits from the intrinsically large étendue of the Michelson interferometer [12] and replaces the moving mirrors by stationary diffraction gratings [11]. In a planar waveguide SHS FT spectrometer, all the outputs of the device, corresponding to different optical path lengths of the interferometer array, are monitored simultaneously in a single measurement of the output spatial interferogram $[\underline{9,10]}$. The source spectrum can be retrieved by mathematical analysis of the interferogram.

In a typical configuration, a waveguide array of MachZehnder interferometers (MZIs) with increasing path differences are used to implement the SHS concept $[9,10]$. For such a geometry, the source power spectrum and the output interferogram are related by the cosine FT. A similar MZI array geometry, including phase-correction circuits using independent heaters for each MZI, has also been demonstrated [13]. However, when long optical path delays are required for high spectral resolution, similar configurations yield prohibitively large devices.

In this Letter, we present a compact FT spectrometer chip, in which a high spectral resolution of $40 \mathrm{pm}$ with a compact device size is achieved by using tightly coiled spiral waveguide structures in an MZI array. Furthermore, a spectral retrieval algorithm with phase and amplitude error compensations is demonstrated for the first time to the best of our knowledge, obviating the need for dedicated phase correction circuits. The FT spectrometer is implemented as an array of $N$ MZIs in silicon-on-insulator (SOI) waveguides (Fig. 1). Each MZI comprises a reference arm of constant length and a delay arm with a spiral waveguide. The length of the delay arm, i.e., spiral length, linearly increases by $\Delta L$ across the array. The high refractive index contrast of the SOI platform and the waveguide bend radius of $\sim 5 \mu \mathrm{m}$ readily allows the making of spirals with geometrical lengths of over a centimeter within an area only a few hundred micrometers in diameter.

For a given input spectral distribution, the dispersive property of the MZI array results in a wavelengthdependent spatial interferogram at the outputs of the array. The relation between the input spectral distribution and the interferogram $I\left(x_{i}\right)$ is unambiguous within

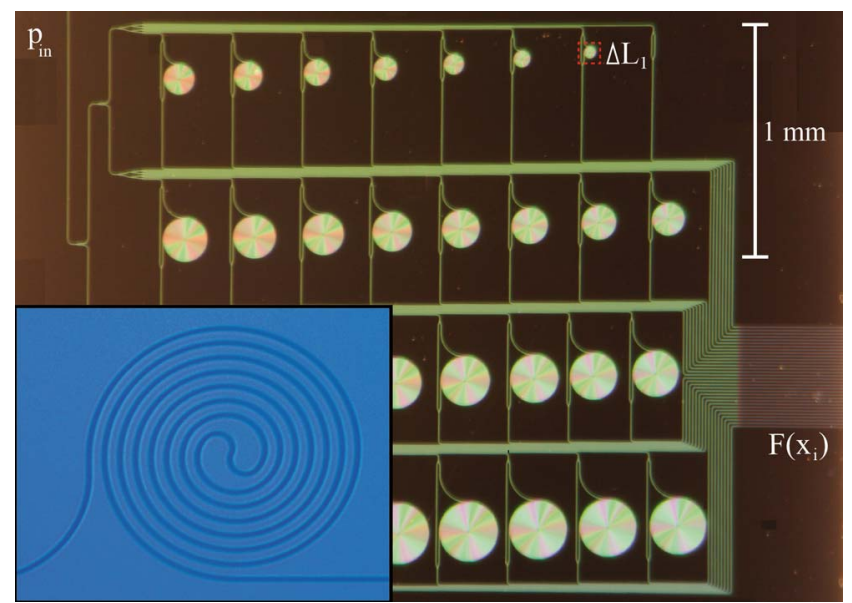

Fig. 1. (Color online) Optical micrograph of the fabricated spatial heterodyne FT spectrometer chip with spiral Si-wire waveguides. Inset: magnified view of the spiral structure. 
the free spectral range (FSR) of an ideal device without phase errors:

$$
I\left(x_{i}\right)=\int_{0}^{\mathrm{FSR}} B(\bar{\sigma}) \cos 2 \pi \bar{\sigma} x_{i} \mathrm{~d} \bar{\sigma},
$$

where $B$ is the incident spectral density, $x_{i}$ is the path delay of the $i$ th MZI, and $\bar{\sigma}=\sigma-\sigma_{L}$ is the shifted wavenumber, relative to the Littrow wavenumber $\sigma_{L}$, at which a monochromatic input produces a constant spatial power distribution at the outputs of the MZI array [10]. The input spectrum can be calculated from the discretized output interferogram using a discrete cosine transform.

The wavelength resolution $(\delta \lambda)$ and the FSR of the device are determined by the maximum path difference and the number of interferometers $[\underline{10}, \underline{13}]$, respectively:

$$
\begin{gathered}
\delta \lambda=\frac{\lambda_{0}^{2}}{\Delta L_{\max } n_{g}}, \\
\text { FSR }=\delta \lambda \frac{N}{2},
\end{gathered}
$$

where $\lambda_{0}$ is the device operational central wavelength and $n_{g}$ is the waveguide group index. The FSR of the device is half the period of the least unbalanced MZI, due to the symmetry of the interferograms.

The FT spectrometer was designed with 32 MZIs and with spiral lengths increasing across the array by $\Delta L=$ $365 \mu \mathrm{m}$ up to $\Delta L_{\max }=1.13 \mathrm{~cm}$ for the most unbalanced interferometer. The longest spiral occupies an area with a diameter of $270 \mu \mathrm{m}$. $450 \mathrm{~nm}$ wide Si-wire waveguides ensure monomode operation and minimize bend losses, with a minimum bend radius of $5 \mu \mathrm{m}$ at the turning point of the spirals. The waveguides were nominally designed for TM polarization, with an effective index of 2.12 at the central wavelength of $1.55 \mu \mathrm{m}$. Our device can be implemented in a layout with $N$ independent input waveguides at a chip facet feeding into the MZI array, for an étendue $N$ times larger than the throughput of a single input waveguide [10]. However, to simplify optical measurements and use conventional telecom waveguide characterization equipment, we used a modified layout with a single input waveguide at the facet followed by cascaded y-couplers. The operational principles and spectral retrieval techniques of both layouts are identical.

Devices were fabricated on SOI with $0.26 \mu \mathrm{m}$ thick silicon and $2 \mu \mathrm{m}$ thick buried oxide. Si-wire waveguide structures were defined in a single patterning step by electron beam lithography using hydrogen silsesquioxane (HSQ) resist. Inductively coupled plasma reactive ion etching was used to transfer the resist pattern into the silicon layer.

The fabricated device was characterized using a highresolution tunable semiconductor laser over the spectral range of 1550-1552 nm, with a wavelength step of $0.1 \mathrm{pm}$. Efficient subwavelength grating couplers [14] were included at the input and output facets of the chip for optimized fiber coupling, while at the same time reducing the Fabry-Perot effect due to the reflectivity at the facets. A Peltier stage was used for thermal stabilization of the chip, and a polarization controller was used to select the TM-polarized input field. Output light from all the MZIs was collected in a single shot with a highsensitivity IR CCD camera.

Figure 2 shows the output interferogram of the 32 MZIs for the measured wavelength range. Detailed inspection of the interferogram reveals two main effects causing deviations from the theoretical performance given by Eq. (1). First, waveguide insertion loss increases with the path difference of the interferometers, resulting in a reduction of the visibility of the MZI transmittance function:

$$
V=\frac{I_{\max }-I_{\min }}{I_{\max }+I_{\min }}=\frac{2 \sqrt{I_{1} I_{2}}}{I_{1}+I_{2}}
$$

where $I_{\max }$ and $I_{\min }$ are the maximum and minimum output intensities of the MZI and $I_{1}$ and $I_{2}$ are the intensities at the outputs of its two arms. Propagation losses of $-4 \mathrm{~dB} / \mathrm{cm}$ were measured, with bending losses of $-1.7 \mathrm{~dB} / \mathrm{cm}$ in the spiral sections. This effect can be readily compensated by dividing the output pattern by the visibility function [Eq. (4)] of each MZI, measured in a calibration wavelength scan. Second, deviations in the effective index and optical path errors arising from fabrication imperfections result in phase errors that distort the interferogram. In particular, a mean group index of 4.38 was measured, with variations of $<1 \%$ in the periodicity of the MZI transmittance across the array from the theoretical (cosine) function. As a consequence of these random variations, the outputs of the MZI interferometers in the array are misaligned in phase and a Littrow wavenumber $\sigma_{L}$ is not found within the analyzed wavelength range. This is a consequence of nonorthogonality of the base of the cosine transform in the presence of phase errors. A solution to incorporate this effect to the model of the discrete Fourier cosine transform was proposed [15]; however, it required phase correction circuits and multiple measurements at varying temperatures.

Here we report a spectral retrieval method that does not require multiple measurements for phase error corrections and also obviates the need for phase tuning. Instead of using the cosine transform, our spectral retrieval algorithm is based on a system of linear equations. The transmittance function of each MZI was sampled at $M$

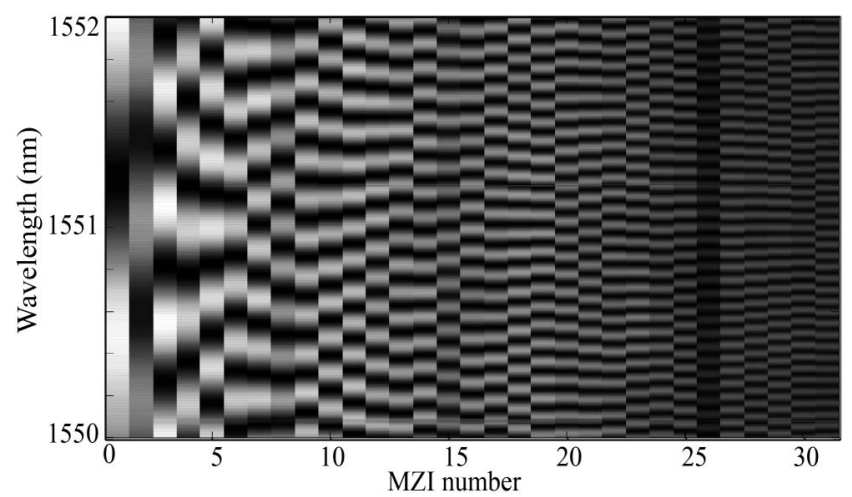

Fig. 2. Experimental measurement of the output power distribution of the 32 MZIs for a $2 \mathrm{~nm}$ wavelength scan near $1.55 \mu \mathrm{m}$ wavelength. 


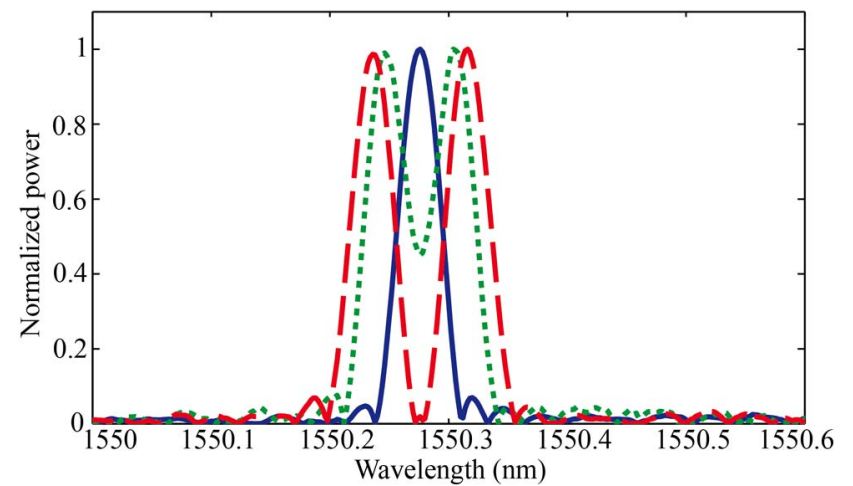

Fig. 3. (Color online) Spectra of a narrowband laser source (solid curve) and two doublets of two monochromatic lines separated by $56 \mathrm{pm}$ (dotted curve) and $80 \mathrm{pm}$ (dashed curve), experimentally retrieved with the spatial heterodyne FT spectrometer chip.

equidistant wavelengths within the FSR of the device. An $N \times M$ transformation matrix $T$ was defined such that $I\left(x_{i}\right)=B \times T$. The source power spectrum $B$ is retrieved by multiplying the spatial interferogram $I\left(x_{i}\right)$ by $T^{+}$, which is a pseudoinverse of the transformation matrix $T$. The pseudoinverse matrix is computed by single value decomposition [16]. In this procedure, the influence of phase and amplitude errors is compensated, since the actual MZI transmittance functions (as measured in the calibration step) are used instead of an ideal cosine function. This yields a robust result compared to the conventional cosine transform technique, which requires an active compensation of the phase errors [15].

Figure 3 shows signal spectra, experimentally retrieved using our device and algorithm, for a single monochromatic source (solid curve), a doublet of two monochromatic lines separated by 56 pm (dotted curve), and a doublet of two monochromatic lines separated by $80 \mathrm{pm}$ (dashed curve). The spectral resolution is $42 \mathrm{pm}$ (measured at FWHM), and the FSR is $0.75 \mathrm{~nm}$. Truncation ripple is reduced by applying an apodization window [17]. Because the matrix $T^{+}$is designed to compensate phase errors of the system, and consequently does not perform an FT of an ideal apodization function without said phase errors, the apodization window is applied directly as a convolution in the spectral domain instead of a multiplication of the interferogram.

It is an important advantage of our device that deviations from the ideal design appear as systematic measurable errors in the interferograms. Once the waveguide device has been fabricated and characterized, the errors can simply be corrected using the spectral retrieval algorithm. This cannot be achieved in devices, such as AWGs, with no direct physical access to the arrayed waveguide output aperture, which makes measuring and correcting phase errors of an AWG a formidable task. Unlike in an AWG, our device provides physical access to each of the arrayed interferometer outputs, where both phase and amplitude errors can be readily measured as part of the spectrometer calibration procedure and compensated in the spectral retrieval algorithm.
We have demonstrated a compact FT spatial heterodyne spectrometer consisting of an array of MZIs with linearly increasing optical path delays. The optical delays are achieved by using Si-wire waveguides arranged in tightly coiled spirals. Spectral retrieval is demonstrated with a wavelength resolution of $40 \mathrm{pm}$ and a FSR of $0.75 \mathrm{~nm}$. The phase and amplitude errors are compensated using a transformation matrix spectral retrieval algorithm. The advantage of software compensation of fabrication errors, along with the high resolution and small footprint of the device, are important practical benefits of this FT spectrometer. Its potential applications include biological and environmental sensing, handheld spectroscopic instrumentation, wavelength-division multiplexing optical channel monitoring, and spectroscopic sensing using space exploration probes or microsatellite platforms.

Financial support from the Spanish Ministry of Economy is acknowledged under grants TEC2008-04105 and TEC2011-23629.

\section{References}

1. P. Cheben, "Wavelength dispersive planar waveguide devices: echelle gratings and arrayed waveguide gratings," in Optical Waveguides: from Theory to Applied Technologies, M. L. Calvo and V. Laksminarayanan, eds. (CRC, 2007), pp. 173-230.

2. P. Cheben, J. H. Schmid, A. Delâge, A. Densmore, S. Janz, B. Lamontagne, J. Lapointe, E. Post, P. Waldron, and D.-X. Xu, Opt. Express 15, 2299 (2007).

3. S. Janz, A. Balakrishnan, S. Charbonneau, P. Cheben, M. Cloutier, A. Delâge, K. Dossou, L. Erickson, M. Gao, P. A. Krug, B. Lamontagne, M. Packirisamy, M. Pearson, and D.-X. Xu, IEEE Photon. Technol. Lett. 16, 503 (2004).

4. J. Brouckaert, W. Bogaerts, P. Dumon, D. Thourhout, and R. Baets, J. Lightwave Technol. 25, 1269 (2007).

5. T. Mizuno, M. Oguma, T. Kitoh, Y. Inoue, and H. Takahasi, IEEE Photon. Technol. Lett. 18, 1570 (2006).

6. F. Xia, M. Rooks, L. Sekaric, and Y. Vlasov, Opt. Express 15, 11934 (2007).

7. P. Bock, P. Cheben, J. Schmid, A. V. Velasco, A. Delâge, S. Janz, D.-X. Xu, J. Lapointe, T. J. Hall, and M. L. Calvo, Opt. Express 20, 19882 (2012).

8. A. V. Velasco, P. J. Bock, P. Cheben, M. L. Calvo, J. H. Schmid, J. Lapointe, D.-X. Xu, S. Janz, and A. Delâge, Electron. Lett. 48, 715 (2012)

9. P. Cheben, I. Powell, S. Janz, and D.-X. Xu, Opt. Lett. 30, 1824 (2005).

10. M. Florjańczyk, P. Cheben, S. Janz, A. Scott, B. Solheim, and D.-X. Xu, Opt. Express 15, 18176 (2007).

11. J. M. Harlander, F. L. Roesler, J. G. Cardon, C. R. Englert, and R. R. Conway, Appl. Opt. 41, 1343 (2002).

12. P. Jacquinot, J. Opt. Soc. Am. 44, 761 (1954).

13. K. Okamoto, H. Aoyagi, and K. Takada, Opt. Lett. 35, 2103 (2010).

14. P. Cheben, P. J. Bock, J. H. Schmid, J. Lapointe, S. Janz, D.-X. Xu, A. Densmore, A. Delâge, B. Lamontagne, and T. J. Hall, Opt. Lett. 35, 2526 (2010).

15. K. Takada, H. Aoyagi, and K. Okamoto, Opt. Lett. 36, 1044 (2011).

16. G. H. Golub and C. Reinsch, Numer. Math. 14, 403 (1970).

17. A. S. Filler, J. Opt. Soc. Am. 54, 762 (1964). 